\title{
Desarrollo de estrategias en el aprendizaje del inglés mediante un juego interactivo: $A$ Journey to Britannia
}

\author{
Development of strategies to teach English \\ through an interactive game: A Journey to Britannia
}

Esperanza Vera Rodríguez
Luz Dary Árias Soto*

Resumen

Este artículo busca socializar los resultados de la exploración del programa $A$ Journey to Britannia, uno de los componentes de la propuesta metodológica diseñada en el marco de la investigación “Exploración de dos propuestas metodológicas hipermediales para el desarrollo de la comprensión auditiva". Para ello, presentamos los principios metodológicos del programa y los resultados obtenidos en este estudio de caso, en relación con las preguntas de investigación: ¿cómo perciben los estudiantes de nivel intermedio de inglés de la Universidad Pedagógica Nacional la propuesta metodológica desarrollada en el juego A Journey to Britannia? Y ¿cómo enfrentan los estudiantes las tareas de escucha?

\section{Palabras clave}

Autonomía, percepción del rasgo, estrategias, propuesta hipermedial, metacognición.

\section{Abstract}

This article is aimed at presenting the results of the exploration of the software A Journey to Britannia, designed within the research project "Exploration of two hyper-medial methodological proposals for the development of listening skills. We will deal with the methodological principles of the software and the results of this case study, which purpose was to answer the following research questions: How do intermediate level students perceive the methodological proposal developed in the interactive game A Journey to Britannia? And how do they face the listening tasks in the program?

Keywords

Autonomy, noticing, strategies, hipermedial proposal, metacognition.

Artículo recibido el 22 de septiembre de 2008 y aprobado el 14 de noviembre de 2008.

* Docentes e investigadoras Departamento de Lenguas de la Universidad Pedagógica Nacional.

Correo electrónico: evera@pedagogica.edu.co y luzdarias@hotmail.com 
En este artículo, presentaremos los principios metodológicos y tecnológicos sobre los cuales se construyó el ambiente hipermedial A Journey to Britannia, entre los cuales podemos mencionar el desarrollo de estrategias de aprendizaje y del acercamiento a una cultura angloparlante, con miras a fomentar la interculturalidad. De igual manera se presentará la metodología de investigación utilizada y los resultados obtenidos en relación con las dos preguntas de investigación relacionadas con la percepción de los estudiantes acerca del programa y las diversas formas utilizadas por estos para enfrentarse a tareas de escucha en la lengua extranjera.

Cuando diseñamos materiales de una manera analítica y reflexiva, la primera consideración que debemos tener en cuenta es el sistema de valores que subyace al currículo de la institución para la cual vamos a crear esos materiales. Todo currículo tiene implícito un sistema de valores que busca desarrollar (White, 1988). Es por ello que debemos preguntarnos qué propósitos buscamos con la educación que brindamos. Por ejemplo, podríamos explicitar si queremos garantizar que nuestros educandos se apropien del legado cultural de la humanidad; si más bien queremos promover el crecimiento del individuo en una sociedad democrática o si buscamos que nuestros estudiantes sean agentes activos del cambio social. De las respuestas que obtengamos para estos interrogantes dependerá, en gran manera, el énfasis que le demos a nuestra propuesta.

\section{Ubicación de la problemática}

Atendiendo a estas necesidades educativas, el equipo de investigación Hipermedia, evaluación y aprendizaje del inglés, de la Universidad Pedagógica Nacional decidió diseñar y validar un ambiente hipermedial para el aprendizaje del inglés que promoviera el logro de algunas de las metas que hemos mencionado. En el equipo creemos que el aprendizaje de una lengua extranjera implica no sólo la adquisición de un sistema para la expresión del significado, cuya función primaria es la interacción y la comunicación (Richards y Rodgers, 2002); aprender una lengua extranjera implica también conocer la cultura del país donde se habla y desarrollar una conciencia intercultural sin perder la identidad; implica aprender a aprender, desarrollar un espíritu crítico, pero tolerante, y aprender a ser autónomo y responsable de su propio aprendizaje.

Teniendo en cuenta estas intrincadas pero fascinantes tareas, decidimos crear dos programas con características diferentes y a la vez complementarias, los cuales conformarían el ambiente de aprendizaje hipermedial para el desarrollo de la comprensión auditiva. Dicho ambiente tendría en cuenta tanto el aprendizaje individual como el colaborativo; tanto el juego, como pretexto para motivar el desarrollo de la comprensión auditiva, como el trabajo con textos de tipo académico; tanto la evaluación controlada de las interacciones, como la evaluación a mediano plazo de la comprensión de los textos orales y escritos.

\section{Participantes}

La muestra observada fue de diecisiete estudiantes de inglés de nivel intermedio de la Universidad Pedagógica Nacional. Ellos participaron en el pilotaje del juego y contribuyeron al mejoramiento de su parte tecnológica. En el siguiente semestre, los alumnos exploraron la versión mejorada del juego. Algunos de ellos también formaron parte del grupo de nivel avanzado que exploró la otra propuesta metodológica.

Todos los participantes firmaron un formato en el que daban su consentimiento para que los investigadores utilizáramos los datos recolectados en la socialización de los resultados de la investigación. Por razones éticas, utilizamos letras para identificar a los participantes, con el fin de mantener en el anonimato sus identidades.

\section{Preguntas de investigación}

La primera pregunta que queríamos respondernos los investigadores era: ¿Cómo perciben los estudiantes de nivel intermedio de inglés de la Universidad Pedagógica Nacional las dos propuestas metodológicas desarrolladas en el ambiente hipermedial? La segunda era: ¿Cómo se enfrentan los estudiantes de nivel intermedio y avanzado a realizar las tareas de escucha? 


\section{Metodología de investigación}

La presente investigación es de tipo cualitativo y el diseño que se utilizó fue el estudio de caso con observación participativa, dada la naturaleza exploratoria del estudio. Seleccionamos este tipo de investigación y este diseño porque como afirma Merriam (1988: 9), un estudio de casos cualitativo es "una descripción y análisis exhaustivo y holístico de una instancia individual, de un fenómeno o de una unidad social", y lo que buscamos es estudiar a fondo el fenómeno del desarrollo de la comprensión auditiva utilizando las Tecnologías de la Información y Comunicación. Además, de acuerdo con Robert Yin (citado en Arzaluz, 2005), para escoger el estudio de caso como metodología para una investigación, el investigador debe preguntarse por el cómo y el porqué de los eventos y no debe intentar controlar ningún aspecto de la situación. Esta es una metodología que permite hacer una observación directa y recoger datos en situaciones naturales.

Para llevar a cabo dicha exploración, utilizamos básicamente dos instrumentos de recolección de datos, a saber: diarios académicos $(\operatorname{logs})$ y entrevistas que se realizaron al finalizar cada sesión. La observación se realizó durante seis meses, tres en el primer semestre y tres en el segundo. Esta observación de un año proporciona al estudio lo que Freeman (1998) denomina triangulación en el tiempo.

De acuerdo con Robin Fagarty (1994), los logs son diarios académicos que se concentran en los trabajos que los estudiantes hacen en el salón y no en otros aspectos de la vida personal de los mismos, como sí lo hacen los diarios (journals). Las entrevistas se entienden como un diálogo directo con los participantes. Este instrumento es útil para recolectar información con propósitos investigativos o docentes (antes y después de la instrucción) y cuando se requiere una retroalimentación uniforme sobre un tópico específico (Genesse y Upshur, 1996).

La metodología de análisis que se siguió es exploratoria, en la cual el investigador lee muchas veces los datos hasta encontrar temas o patrones recurrentes (Freeman, 1998). Para garantizar la validez interna de los resultados, se usaron varios instrumentos de recolección de datos y se trianguló la información que se encontró en los diferentes instrumentos. Como afirma Faltis (1997) algunas de las formas más populares para establecer la validez interna en una investigación de estudio de caso son la triangulación, la observación prolongada en el sitio que se investiga el caso, el análisis y la revisión reiterada de las afirmaciones de los participantes y las suposiciones y orientaciones teóricas conocidas desde el comienzo del estudio.

El equipo de investigadores se dividió en subgrupos, a cada uno de los cuales se le asignó una tarea: hacer entrevistas, transcribirlas, administrar los logs y transcribirlos. Esto garantizaba la triangulación de investigador (Freeman, 1998). El análisis de cada una de la entrevistas se hizo individualmente, buscando temas que permitieran inferir la percepción de los estudiantes acerca de las dos piezas de software y determinar qué estrategias reportaban haber utilizado para resolver las tareas de escucha. Luego cada co-investigador puso en común en el equipo los temas encontrados, esto con el fin de clasificar los datos en los patrones que emergieron de la puesta en común, para posteriormente reducir los datos, diagramar relaciones entre los patrones y configurar las categorías.

Tanto para los datos de las entrevistas, como para los datos de los logs, se asignaron códigos de colores, de acuerdo con las preguntas de investigación. Además, se asignaron números y letras a los patrones que iban surgiendo. A medida que se analizaban los datos, éstos se agrupaban en categorías y subcategorías de acuerdo con la frecuencia de aparición de cada una ellas. Esta metodología de triangulación del análisis obtenido por los investigadores y los diferentes tipos de instrumentos le da validez a los resultados obtenidos.

\section{Marco teórico que sustenta el diseño del juego interactivo}

En la parte metodológica, los docentes investigadores le apostábamos con esta propuesta a la integración de habilidades, al entrenamiento en estrategias de aprendizaje, al desarrollo de la competencia intercultural y a la promoción del trabajo autónomo como herramientas para apoyar el desarrollo de la 
comprensión auditiva. En la parte tecnológica, le apostamos a la interactividad, a la retroalimentación, al poder del juego como elemento motivador en el aprendizaje, a la fascinación que producen las leyendas; al poder de la imagen para fijar información adquirida mediante otros modos de comunicación, a la evaluación inmediata que puede realizar el programa, así como a la variedad de escenarios, personajes y situaciones comunicativas que el usuario debe comprender para poder "interactuar" con los personajes de manera adecuada.

Este material digital está fundamentado en un enfoque que combina lo cognitivo y lo lúdico del aprendizaje en un juego interactivo enmarcado en la época del joven Rey Arturo y el gran mago Merlín, en el siglo VI. El juego consta básicamente de dos módulos: uno de entrenamiento y práctica de las estrategias cognitivas específicas para la habilidad de escucha y otro que contiene las dos grandes misiones que el usuario debe llevar a cabo para poder regresar al siglo XXI, con sus compañeros de excursión en Gran Bretaña. El primer módulo contiene siete juegos relacionados con cada una de las estrategias, que se pueden visitar en cualquier orden y que el usuario debe obtener un ochenta por ciento de aciertos para poder acceder al segundo módulo.

El segundo módulo contiene el gran juego de roles, en el cual el usuario interactúa con los personajes de una manera simulada, al escoger la mejor de cuatro opciones de respuesta. Si la interacción escogida es correcta, el usuario gana una vida y continúa relacionándose con los personajes y las situaciones fantásticas; de lo contrario, el usuario pierde vidas, pero puede seguir jugando hasta que las haya perdido todas. Toda la información del usuario queda almacenada en el perfil del usuario, creado desde el inicio del juego y que está disponible cada vez que el usuario termina una misión.

Al diseñar el juego interactivo, tuvimos en cuenta ciertos principios pedagógicos y didácticos relacionados con la potencialidad pedagógica del juego, la comprensión de textos orales, la potencialidad del uso de estrategias de aprendizaje, al igual que el conocimiento de elementos de una cultura y la inmersión en uno de sus productos, como son sus leyendas.

\section{La potencialidad del juego}

Piaget afirmaba en 1930 (citado en Caillois, 1997) que uno de los valores del juego en la formación moral del niño es el del respeto por sus reglas. De manera general, el juego aparece como educación del cuerpo, del carácter o de la inteligencia, ya que ayuda a desarrollar aptitudes y cuanto más se aleje de la realidad, mayor es su valor educativo.

Para Vigotsky, el juego suele ser la principal actividad del niño, además de una de las maneras de hacer participar al niño en la cultura. En el juego existe una estricta subordinación a ciertas reglas que no son posibles en la vida real; de esta forma, el juego crea una Zona de Desarrollo Próximo en el niño porque supone la creación de una situación imaginaria regida por determinadas reglas de conducta.

Para Vigotsky, (citado en Baquero, 1996) debe considerarse, además, la amplitud del juego en cuanto a los cambios de acuerdo con las necesidades de los niños.

Creemos que el uso de los juegos potencia cualquier aprendizaje, ya que éstos, además de incrementar la motivación intrínseca en aprendices de todas las edades (Brown, 1994), contienen los mismos elementos de un problema y requieren del uso de estrategias de solución de problemas. En el primer software, los estudiantes deben utilizar sus competencias para poder interactuar apropiadamente en el juego construido, el cual se desarrolla en torno a la historia fantástica ya mencionada. Estas características de los juegos, junto con las estrategias cognitivo-linguísticas que se trata de fomentar, pueden convertirse en herramientas cognitivas que le permitan al estudiante aprender con el computador y no del computador, y así entrar en "compañerismo" con éste, de tal manera que en vez de ser controlado por él, como afirma Jonassen (2002), "el estudiante pueda resaltar la capacidades del computador y el computador pueda resaltar su pensamiento y aprendizaje".

\section{Procesos en la comprensión de textos orales}

Dado que la comprensión auditiva en inglés es una de las actividades que causa mayor dificultad a nuestros aprendices y conociendo que la tecnología 
puede desempeñar un papel importante en la solución de problemas de aprendizaje y contribuir al desarrollo de la autonomía, se decidió proponer una innovación pedagógica acorde con esas necesidades. Dicha innovación es un ambiente hipermedial para el desarrollo de la comprensión auditiva en inglés, del cúal hace parte esta propuesta metodológica hipermedial que buscan proporcionar al aprendiz diferentes escenarios y diferentes tipos de actividades pedagógicas que involucran el uso de diversos medios, como audio, texto, fotografía, animaciones y gráficas para potenciar el aprendizaje.

En la comprensión de lo que escuchamos en una lengua extranjera, L2, intervienen una serie de procesos psicomotrices, linguísticos, paralinguísticos y sociales que se entretejen y hacen de esa habilidad una de las más difíciles de desarrollar en el proceso de aprendizaje de esa L2. ¿Qué hace que la comprensión de textos orales sea difícil?

De acuerdo con Douglas Brown (1994), hay ocho elementos que hacen que la comprensión del discurso oral se dificulte a los hablantes no nativos de una lengua, pero que si son conocidos pueden ser utilizados por el oyente para agudizar su comprensión. Algunos de esos elementos son: la redundancia, es decir, aquellas repeticiones o reelaboraciones que hacemos a medida que hablamos; el uso de formas reducidas al hablar; las variables que afectan la actuación en el discurso oral no planeado; el lenguaje coloquial, expresiones idiomáticas, jerga y conocimiento cultural compartido por los hablantes y la velocidad con que se produce el discurso oral, aunque autores como Jack Richards (1990) han señalado que el problema radica más en el número y la duración de las pausas que hacemos los hablantes nativos que en la velocidad con que se produce el discurso.

Es importante también mencionar que tuvimos en cuenta los principios para diseñar técnicas de escucha derivados de décadas de investigación y práctica. Los dos primeros se pueden aplicar a cualquiera de las habilidades; los otros son más característicos de la escucha. Brown (1994) sugiere que debemos asegurarnos de no descuidar la importancia de las técnicas que desarrollan específicamente la compe- tencia para comprender la escucha, que éstas sean intrínsicamente motivantes, que requieran tanto de procesamiento ascendente como descendente y que utilicen lenguaje auténtico y contextos de comunicación. Además, recomienda tener en cuenta que las respuestas de los oyentes indiquen si han comprendido de manera correcta y que se fomente el desarrollo de estrategias de escucha.

Por tanto, conscientes de la dificultad que implica para la mayoría de los aprendices comprender a los hablantes nativos de esta lengua y de la variedad de micro-habilidades que es necesario fomentar para ello, planteamos dos diseños conceptuales diferentes para abordar los dos tipos de comprensión auditiva planteados por Anderson y Lynch (citados en Brown, 1994), recíproca y la no recíproca. Este software promueve la comprensión recíproca, es decir, aquella en la que el oyente tiene la oportunidad de interactuar con los hablantes, que en nuestro caso son virtuales.

\section{La potencialidad del}

\section{entrenamiento en estrategias}

Pedagógicamente, la propuesta se sustenta en la teoría cognitiva y en el constructivismo. Para el diseño de este software nos apoyamos en un enfoque cognitivo, por cuanto consideramos que el aprendiz debe involucrarse activamente en su aprendizaje, el cual implica no sólo aprender un conocimiento declarativo (el qué), sino que, además, es necesario adquirir un conocimiento procedimental (el cómo) que permita procesar, organizar, almacenar y utilizar el conocimiento declarativo constituido por hechos, datos, reglas, historias, entre otros elementos (Anderson, citado en Gagné, 1985).

1. En el ámbito del conocimiento procedimental, como ya lo mencionamos, se encuentran las estrategias de aprendizaje. Rebecca Oxford (1991) presenta doce rasgos de estas estrategias que hacen posible el desarrollo de una competencia comunicativa en el aprendiz, las cuales contribuyen a desarrollar aspectos particulares de la competencia comunicativa, a saber: las competencias gramatical, sociolingüística, discursiva y estratégica; permiten a los estudiantes ser más 
autodirigidos; expanden el papel del profesor; están orientadas hacia la resolución de problemas; son acciones específicas tomadas por el aprendiz; involucran otros aspectos del aprendiz, no sólo lo cognitivo; apoyan el aprendizaje directa e indirectamente; a menudo son conscientes; pueden ser enseñadas; son flexibles y son afectadas por una variedad de factores (estadio de aprendizaje, requerimientos de la tarea, estilo de aprendizaje general, personalidad, motivación y el propósito para aprender una lengua, etc.).

\section{La interacción como}

\section{factor clave en el aprendizaje}

De acuerdo con la tesis de Vygotski (citado en Barraza, 2001), el aprendizaje es un proceso de construcción del sujeto que establece una relación de sucesión entre lo interpersonal y lo personal; es primero interpersonal y luego se convierte en personal, por medio de la apropiación que hace el sujeto del conocimiento. Así, las relaciones intersubjetivas influyen en la relación del sujeto con el objeto de conocimiento. Los procesos de enseñanza y de aprendizaje que se desarrollan en ámbitos institucionales están signados por la intencionalidad social, mediada por la acción del docente, lo que hace posible entender el papel del maestro por medio del concepto de mediación intersubjetiva.

Para llevar a efecto esta función, el docente puede auxiliarse de diferentes medios, de carácter situacional, instrumental o lingüístico, siendo este último rasgo el que conduce al concepto de mediación semiótica, el cual constituye una extensión del sentido original que le dio Vygotski al concepto en su último giro teórico y que James Wertsch ha denominado mediación semiótica y ha venido popularizando desde 1980. Aunque estas dos acepciones conducen a aspectos específicos diferentes no son excluyentes, se pueden complementar. La mediación semiótica se da mediante diferentes instrumentos o herramientas culturales, tal y como afirma Barraza Macías (2001) en su documento "Constructivismo social: un paradigma en formación": citando a Vygotski "la lengua, las diferentes formas de numeración y cálculo, los mecanismos mnemotécnicos, los simbolismos alge- braicos, las obras de arte, la escritura, los esquemas, los diagramas, los mapas, los dibujos y toda clase de signos convencionales". Estamos de acuerdo con Barraza Macías en que todos estos ejemplos son creaciones artificiales de la humanidad, que hacen parte de la cultura y que tienen sobre todo una función de representación, lo que hace que James Wertsch denomine el proceso que realizan como mediación semiótica. La forma de mediación semiótica que adquiere mayor relevancia para el aprendizaje es el lenguaje, importancia que el mismo Vygotski le reconoce en la formación del concepto.

En la propuesta que subyace a este software, utilizamos diferentes tipos de instrumentos psicológicos de mediación, como el lenguaje oral y escrito, que buscan promover la interacción mediante una simulación de ese proceso, en el cual nuestra mediación como docentes se da por medio de la retroalimentación, que se proporciona cuando el estudiante escoge una opción de interacción inapropiada, ya que se le informa qué tipo de error ha cometido, para que así pueda desarrollar un proceso de metacognición, para aprender de ese error. Otros instrumentos de mediación semiótica utilizados en este juego interactivo son los dibujos de los personajes y las fotografías de sitios turísticos de Inglaterra que se presentan al usuario antes de iniciar las misiones, con el fin de ayudarle a formarse una representación de la cultura en la que se va a mover en el juego.

\section{La potencialidad de la hipermedia}

Antes de discutir la potencialidad de la hipermedia, es importante definir el término. Como menciona Lamarca (2006) en su tesis doctoral, Jakob Nielsen, en "Hypertext and hypermedia" (1990), establece la diferencia entre hipertexto e hipermedia, desde su punto de vista, cuando explica que el hipertexto consiste en piezas de texto o de otro tipo de presentación de la información ligadas de manera no secuencial, mientras que si el foco de tal sistema descansa en tipos de información no textual, se utiliza el término hipermedia.

Estamos de acuerdo con la definición de hipermedia hecha por Nielsen, pero añadiríamos que la inclusión de diferentes medios para representar la 
información (audio, texto, imágenes, animaciones, representaciones gráficas) nos permite además promover los diferentes modos de acceder a ella, concepto que se conoce como multimodalidad, y del cual se afirma que potencia el aprendizaje. La práctica con ellos, la variedad de recursos disponibles (diccionarios, posibilidad de crear archivos de texto y enviarlos a un correo electrónico) y los diferentes tipos de actividades que se pueden incluir en programas de aprendizaje mediado por computador nos permiten atender las necesidades de estudiantes con diferentes estilos cognitivos, proporcionar retroalimentación inmediata, realizar un trabajo personalizado en grupos grandes, tener la facilidad de realizar trabajo por proyectos por pares o grupos pequeños, bien sea colaborativa o competitivamente, incluir el factor lúdico; fomentar un aprendizaje exploratorio a partir del análisis de corpus lingüístico y promover el desarrollo de habilidades computacionales, además de proporcionar herramientas que faciliten el procesamiento y la representación del conocimiento.

\section{Resultados de la investigación}

En este apartado se presentarán los resultados, describiendo las categorías que emergieron de los datos y que responden a cada una de las preguntas de investigación e incluyendo palabras textuales de los participantes para sustentar cada una de las categorías y subcategorías. A continuación, presentamos las categorías que emergieron como respuesta a cada una de las preguntas de investigación. Cada grupo de estrategias que compone las categorías, será sustentado utilizando palabras textuales de los participantes, tomadas de los logs y de las entrevistas, añadiendo la fecha en que fueron realizadas y utilizando letras para identificar a los participantes, con el fin de mantener su identidad en el anonimato, como fue convenido en el formato de consentimiento.

Pregunta 1 ¿Cómo perciben los estudiantes la propuesta metodológica A Journey to Britannia, desarrollada en el ambiente hipermedial?

\begin{tabular}{ll}
\hline Categoría & Subcategorías \\
\hline & $\begin{array}{l}\text { La guía que proporcionan los } \\
\text { programas (instrucciones, } \\
\text { explicaciones, ejemplos). }\end{array}$ \\
\cline { 2 - 2 } \begin{tabular}{l} 
1. Instrumento de práctica \\
contextualizada e interactiva contextualizada \\
que genera motivación y \\
facilita la comprensión. \\
\cline { 2 - 2 } $\begin{array}{l}\text { (contexto lingüístico y } \\
\text { situacional) e interactiva. }\end{array}$
\end{tabular} & $\begin{array}{l}\text { El diseño gráfico de las } \\
\text { propuestas (imágenes, mapas, } \\
\text { actividades). }\end{array}$ \\
\cline { 2 - 2 } & $\begin{array}{l}\text { El contacto con textos orales } \\
\text { adecuados y con diferentes } \\
\text { acentos (audio). }\end{array}$ \\
\hline $\begin{array}{l}\text { 2. Instrumento para la } \\
\text { adquisición de conocimiento } \\
\text { y desarrollo de habilidades. }\end{array}$ & $\begin{array}{l}\text { Adquisición de conocimiento } \\
\text { lingüístico y paralingüístico. }\end{array}$ \\
\cline { 2 - 2 } $\begin{array}{l}\text { 3. Instrumento que promueve } \\
\text { el trabajo autónomo y la integral de } \\
\text { reflexión. }\end{array}$ & habilidades. \\
\hline
\end{tabular}

Al tratar de dilucidar qué características de los programas permitieron que los estudiantes evaluaran el ambiente hipermedial en términos positivos, los estudiantes aseveran que esta es una metodología novedosa caracterizada por los aspectos que se enuncian a continuación y que constituyen las categorías y subcategorías que responden a la primera pregunta.

\section{Es un instrumento de práctica contextualizada e interactiva que genera motivación y facilita la comprensión}

A partir del análisis de datos, pudimos encontrar que los estudiantes percibieron las propuestas como una forma didáctica, fácil, diferente y dinámica de aprender, como se evidencia en los siguientes ejemplos:

E: $Y$ en términos generales, ¿qué concepto del juego tienes?

T: No, pues es bueno, es bien, me gustó, es aplicable, es didáctico, interactivo, dinámico y si se maneja bien, da resultados (entrevista del 11 de mayo de 2004).

Los patrones relacionados con esta categoría tienen que ver con las características de los programas que no sólo despiertan el interés de los estudiantes, sino que también los motivan a usarlos para mejorar 
su comprensión auditiva, sin descuidar otras habilidades. Una de esas características es la guía que proporcionan los programas al usuario.

\section{a. La guía que proporcionan los programas (instruc- ciones, explicaciones, ejemplos)}

Según los estudiantes, tanto las explicaciones como los ejemplos e instrucciones de las propuestas les permiten lograr una mayor comprensión de los juegos y las actividades y los ayudan, por ende, a alcanzar un mejor desempeño. De la misma manera lo hace la interactividad lograda mediante la retroalimentación que permite al estudiante evaluar su desempeño y reformular aquellas respuestas que no sean apropiadas, fomentando el proceso de metacognición, eje de estas propuestas hipermediales. Esto se puede ver en el siguiente comentario:

E: Bien, ¿qué te llamó más la atención de las misiones, fuera de la trama?

D: Um, pues un poco el contexto, pero también como el vocabulario que se usa, eh, que es uno seguir mucho las instrucciones (entrevista del 26 de noviembre).

Con referencia a las explicaciones, los estudiantes las consideraron adecuadas para comprender los juegos y desarrollarlos.

E: ¿Qué te resultó útil?

C: ¿Útil? Las explicaciones que dan al principio antes de jugar, o digamos, por ejemplo, cuando era atrapar la idea rápido, le daban a uno unos ejemplos y buscar la respuesta, pero mentalmente. Entonces, eso lo ayuda a uno mucho como para ir formándose cómo va a ser el juego después (log del 4 de mayo, pregunta 3).

En relación con los juegos, los estudiantes comentaron que A Journey to Britannia es una forma lúdica e interesante de trabajar la práctica y la teoría, además de ser una posibilidad de aprendizaje creativo.

MD: Yo pienso que los juegos están completamente relacionados con la teoría. Y si estuvieran los juegos aparte de la teoría, pues sería imposible porque no habría un conocimiento previo para poder desarrollar el juego (entrevista del 11 de mayo).

\section{b. La práctica contextualizada (contexto lingüístico $y$ situacional) e interactiva}

Otra característica de los programas que llamó la atención de los estudiantes fue la posibilidad de interactuar con personajes del juego, de aprender en un contexto y recibir retroalimentación en las dos propuestas. Esto les permitió tener oportunidades para realizar una práctica interactiva y contextualizada, como ya hemos mencionado.

Una de las características de A Journey to Britannia en las dos misiones del juego era que proporcionaba evaluación inmediata de las respuestas al estudiante, pero, además de esto, le daba pistas de tipo pragmático mayormente sobre el tipo de error que había cometido al escoger cualquiera de los distractores incorrectos. Un gran número de estudiantes consideró que es un software que les da la oportunidad de realizar una práctica interactiva, ya que les van dando información sobre sus aciertos y porque, además de tener que interactuar con los personajes de la historia, el usuario recibe evaluación inmediata de sus respuestas y retroalimentación sobre las posibles razones por las cuales sus respuestas podrían estar equivocadas. Al preguntarles a los estudiantes qué les llamó la atención del juego o qué les resultó útil respondían con afirmaciones como las siguientes:

YM: [...] De pronto el juego me devolvía, volvía y si colocaba la respuesta el computador, ahí si me daba cuenta si lo que yo había hecho de pronto estaba mal o estaba bien y por qué. Pero entonces no era colocar la respuesta y seguir como si nada, pero volver a retomar (entrevista del 11 de mayo).

Como afirman Warschauer y Healey (1998), el progreso en el desarrollo de habilidades específicas depende, en gran medida, de la clase y la calidad de la retroalimentación que se ofrece; dicha calidad personaliza la educación y permite mejores resultados individuales. El mejoramiento en los resultados, por lo general, se traduce en un aumento en el grado de interés y, en consecuencia, en el deseo de participar.

Además de la interacción comunicativa y la interactividad entre el programa y el usuario, algunos 
estudiantes señalaron la interacción que se puede establecer entre la teoría y la práctica, tal como aparece en una de las respuestas de la entrevista.

E: Bueno, eh, terminando el juego ¿qué opinión general tienes sobre él y el final?

MD: Eh, fue un juego muy productivo para mí, eh, me gustó mucho, me gustó mucho cómo interactuaron la teoría con la práctica, eh... fueron como, no sé, trabajos diferentes, o sea teníamos que, que llegar a una meta, pero esa meta no iba sola, teníamos que desarrollar nuevas habilidades, nuevas cosas que pues obviamente nos van a servir para después (entrevista del 11 de mayo).

c. El diseño gráfico de las propuestas (imágenes, actividades)

Otra característica del programa que motivó a los estudiantes fue el diseño de la propuesta.

E: Una vez que terminaste ya tu juego, ¿qué opinión tienes en general de él?

LG: El tema me pareció muy interesante, los ejemplos, el diseño de la presentación es excelente, junto con el diseño de juegos (entrevista del 23 de septiembre).

d. El contacto con textos orales adecuados, cotidianos $y$ con diferentes acentos

En relación con los tipos de textos orales con los que tuvieron contacto los estudiantes en el juego interactivo, debemos mencionar en primer lugar que los estudiantes consideraron que éste les permitió escuchar textos orales adecuados a su nivel. Ejemplo:

Para mi nivel de lengua el inglés utilizado fue...

FV: El adecuado para nosotros; además, todos los hablantes son nativos, esto ayuda a mejorar las estrategias al escuchar ( $\log$ del 26 de octubre, pregunta 7).

En segundo lugar, los estudiantes opinaron que el juego les permitió el contacto con lenguaje cotidiano y con diferentes acentos. Ejemplo:

E: ¿Qué tal los contenidos?

CM: Pues sí son como muy aplicables por la cotidianidad que no se ve de pronto en los libros, ¿sí?, los libros son muy formales, muy estructurados y esto me parece que es más cotidiano, más de lo que uno utiliza afuera ya en el aula común y corriente (entrevista del 11 de mayo).

Sólo dos estudiantes opinaron que el nivel de lengua utilizado en A Journey to Britannia era complejo. Ante la pregunta ¿Cómo te pareció el nivel de lengua de los hablantes?, uno de ellos respondió:

MD: Un poquito complejo, un poquito complejo, la verdad. Porque tienden a hablar muy rápido. $\mathrm{Y}$ entonces, como ya le había comentado, profesora, esta es la habilidad más complicada para mí, entonces no sé. Personalmente creo que hablaban un poquito rápido, entonces me perdía en algunas cosas (entrevista del 11 de mayo).

Además de generar motivación en los usuarios y facilitar la práctica interactiva y contextualizada, los estudiantes percibieron que la propuesta les permitía adquirir diferentes tipos de conocimiento y les ayudaba a desarrollar habilidades para el mejor desempeño en el uso del inglés. A continuación, presentamos la categoría y las subcategorías que emergieron de patrones relacionados con esta percepción.

\section{Instrumento para la adquisición de conoci- miento lingüístico y paralingüístico y el desa- rrollo de habilidades}

Los patrones que permitieron configurar esta categoría tienen relación con los conocimientos que los estudiantes adquirieron con el uso de los programas, no sólo en cuanto a aspectos culturales de Inglaterra, sino también en cuanto al dominio de la lengua inglesa. En relación con la adquisición de conocimiento lingüístico y paralingüístico, los estudiantes consideraron que el programa les ofrece la oportunidad de escuchar un discurso real, con diversos acentos y acorde con su nivel de lengua. Asimismo, según las opiniones de algunos de los usuarios, el trabajo con las propuestas contribuyó específicamente al mejoramiento de su comprensión auditiva y, además, según otros estudiantes, les permitió desarrollar de manera integrada otras habilidades como la comprensión lectora, la escritura e incluso la comunicación oral. 
a. La propuesta facilita la adquisición de conocimiento lingüístico y paralingüistico

Veamos un ejemplo relacionado con la adquisición de conocimiento lingüístico:

MD: Aprendí nuevo vocabulario porque había cosas que realmente no había visto, entonces siempre fue muy bueno por eso; además por lo que ya mencioné que era la parte del listening, que para mí es importante (entrevista del 26 de noviembre).

En relación con el conocimiento paralingüístico, los estudiantes afirmaron haber aumentado sus conocimientos sobre acentuación, tono, entonación, ritmo. Veamos un ejemplo:

En el día de hoy aprendí:

DR: Varios aspectos que no conocía, pero que son de vital importancia para un mejor aprendizaje como son reasoning deductively, dissapearing consonants, sentences stress, pitch, etc (log del 4 de mayo, pregunta 2).

Los datos anteriores nos permiten observar que los estudiantes son conscientes de las formas lingüísticas que se les presentan en la propuesta metodológica A Journey to Britannia, lo que les permite apropiarse de nuevos conocimientos, según la hipótesis del reparo o la percepción del rasgo (del inglés noticing).

Para Schmidt (1995), quien fue el primero en hablar de la hipótesis de la percepción del rasgo, dicha percepción es útil para que el aprendiz tenga un mejor desempeño en el manejo de la lengua. Según esta hipótesis, lo que el aprendiz "nota" en la información (input) que recibe es lo que se convierte en objeto de aprendizaje.

Aparte de ser un programa que les ayuda a adquirir conocimientos, para algunos estudiantes la propuesta ofrece la oportunidad de profundizar conocimientos ya adquiridos o reforzarlos. Ejemplo:

LQ: Es una manera muy divertida e interactiva de fortalecer cosas que de pronto uno no sabe, o que ya sabe y necesita fortalecer (entrevista del 26 de noviembre).

b. Facilita el desarrollo integral de habilidades Un gran número de estudiantes coincidió en afirmar que con el uso del programa su habilidad de escucha en la lengua extranjera mejoró en gran medida. Asimismo, algunos consideraron que el trabajo con la propuesta les ayudó a desarrollar e integrar otras habilidades, es decir, que aunque ésta centra su objetivo en el desarrollo de la comprensión auditiva, también permite desarrollar la comprensión de lectura y la escritura en menor grado. Ejemplo:

MD: Britannia me ha servido mucho sobretodo para el trabajo de listening en el laboratorio; entonces pues de ya alguna manera uno, eh, empieza a desarrollar mejor ese sentido del oído, y en el laboratorio me ha servido mucho (entrevista del 26 de noviembre).

Además de la motivación por el aprendizaje y la adquisición de conocimientos, los estudiantes perciben que los programas los ayudan a desarrollar características del trabajo autónomo.

\section{Es un instrumento que promueve el desarrollo de la autonomía}

La propuesta proponía a los estudiantes diferentes estrategias de aprendizaje para enfrentarse a las tareas. En el juego interactivo se presentaba un tipo de trabajo autónomo de aplicación de estrategias de aprendizaje que habían sido modeladas en un módulo previo al juego mismo. $\mathrm{Al}$ analizar los datos, encontramos que muchos estudiantes reportaron haber utilizado algunas de las estrategias que se habían modelado y mostraron indicios de estar haciendo metacognición sobre sus procesos de aprendizaje.

E: Esas estrategias que tú mencionas, y las que mencionaste antes, eh, ¿forman ahora parte de tus esquemas mentales, o ya las habías utilizado antes en otros ejercicios?

F: No, pues yo nunca había interactuado con algo así por el estilo, nunca en ningún lado, en ningún estudio, entonces, eh, a partir de las estrategias, pues, es que lo que pasa es que yo escribí las estrategias en un cuaderno...

En esta propuesta metodológica, se le apostó al uso de estrategias de aprendizaje, con el fin de incentivar el trabajo autónomo en el estudiante. El equipo investigador coincide con Holec (1980) 
en que el concepto de autonomía en la enseñanza comunicativa de la lengua se define como la capacidad que tiene el estudiante de controlar su propio aprendizaje, lo que lo lleva ser independiente y capaz de resolver los problemas que se presenten en su aprendizaje.

Consideramos que para lograr que el estudiante sea autónomo, se le debe proporcionar instrucción directa acerca de ciertas estrategias para aprender la lengua y hacerlo consciente de cuándo y cómo debe usarlas para que pueda convertirse en un estudiante independiente. Esta, según Tudor (2001: 119), es la etapa de entrenamiento, en la cual se ayuda al estudiante, mediante un proceso de aprendizaje de una manera autodirigida, lo cual se evidencia en Britannia. Podemos concluir diciendo que la percepción general de los estudiantes es que A Journey to Britannia es una forma didáctica y motivante de aprender. El engranaje metodológico-tecnológico del software promueve la adquisición de conocimiento, el desarrollo de habilidades y el trabajo autónomo.

Pregunta 2 ¿Cómo se enfrentan los estudiantes de nivel intermedio y avanzado a realizar las tareas de escucha?

\begin{tabular}{|c|c|}
\hline Categoría & Estrategias \\
\hline \multirow{3}{*}{$\begin{array}{l}\text { 1. Lectura analítica y } \\
\text { toma de notas. }\end{array}$} & Leer cuidadosamente para entender la tarea requerida. \\
\hline & $\begin{array}{l}\text { Establecer relaciones entre la información oral específica y las } \\
\text { opciones de respuesta. }\end{array}$ \\
\hline & Tomar notas para completar una tarea. \\
\hline \multirow{5}{*}{$\begin{array}{l}\text { 2. Fijación de la atención } \\
\text { en información textual y } \\
\text { contextual específica. }\end{array}$} & $\begin{array}{l}\text { Centrar la atención en información clave (tema, palabras } \\
\text { clave, adjetivos, información nueva-dada, sufijos y prefijos). }\end{array}$ \\
\hline & Identificar el contexto situacional o semántico. \\
\hline & Hacer inferencias con base en el contexto. \\
\hline & Analizar el registro del lenguaje (formal o informal). \\
\hline & $\begin{array}{l}\text { Establecer asociaciones (para formar grupos semánticos y } \\
\text { aprender vocabulario). }\end{array}$ \\
\hline \multirow{4}{*}{$\begin{array}{l}\text { 3. Uso de procesos } \\
\text { metacognitivos. }\end{array}$} & Escuchar varias veces para confirmar la comprensión auditiva. \\
\hline & Planear y monitorear el proceso de aprendizaje. \\
\hline & Aprender de los errores como herramienta de aprendizaje \\
\hline & $\begin{array}{l}\text { Usar otros recursos para verificar la comprensión (diccionario, } \\
\text { la profesora). }\end{array}$ \\
\hline \multirow{3}{*}{$\begin{array}{l}\text { 4. Aplicación de } \\
\text { conocimientos previos } \\
\text { y nuevos. }\end{array}$} & Entender rasgos de pronunciación y suprasegmentales. \\
\hline & $\begin{array}{l}\text { Usar conocimiento declarativo lingüístico y no lingüístico } \\
\text { previo y nuevo. }\end{array}$ \\
\hline & Utilizar reglas fonéticas. \\
\hline
\end{tabular}

Analizando la información recolectada para responder la segunda pregunta, encontramos que los estudiantes recurren a diversas formas de enfrentarse a dichas tareas. Hemos decidido llamar a dichas formas "estrategias", ya que parecen ser formas personales de aproximarse a la resolución de una tarea, con diferentes grados de eficiencia. Estas estrategias fueron agrupadas en categorías mayores, de acuerdo con las similitudes y la frecuencia que presentaron. A continuación, presentamos las cate- gorías que emergieron a partir del análisis cuidadoso y repetitivo de los datos, ordenadas de las más a las menos frecuentes. Veamos ahora algunos ejemplos que dan sustento a las categorías y subcategorías que emergieron del análisis y reducción de los datos.

\section{La lectura analítica y la toma de notas}

Las estrategias agrupadas en esta categoría son todas de carácter cognitivo. Entre ellas tenemos: 
a. Leer cuidadosamente para entender la tarea requerida

Las opiniones de algunos estudiantes nos llevan a pensar que el éxito de los alumnos para realizar las tareas radica en el hecho de entender muy bien las instrucciones y lo que deben hacer antes de lanzarse directamente a interactuar con los juegos y con los ejercicios. El siguiente ejemplo ilustra esta estrategia:

MD: Cuando yo leía la teoría, miraba los ejemplos $y$ trataba de fijarme mucho en la estructura y como en el significado de cada una de las palabras, de manera que cuando ya estaba en el juego, yo hacía lo mismo, miraba las palabras y los ejemplos y de acuerdo a eso podía responder la pregunta que le hacían en los juegos. Entonces, básicamente esa fue mi estrategia. Mirar y comprender bien los ejemplos. Ante todo la teoría, mirarla bien para poderla aplicar en los juegos (entrevista del 11 de mayo).

b. Establecer relaciones entre la información oral específica y las opciones de respuesta

Esta fue otra estrategia de alta frecuencia, ya que cuando los usuarios escuchaban los ejercicios de audio, se les formulaban preguntas de comprensión en forma de opción múltiple para que pudieran comprender las situaciones, la historia del juego principal e interactuar con los personajes de cada misión.

CM: ¿Qué más apliqué? Antes de... digamos que mientras iba escuchando iba leyendo las opciones. Como que uno va desechando unas que no tienen nada que ver. O sea, trata uno como de predecir qué es lo que está diciendo el rey. Entonces trata uno como de predecir, y de ir asociando con las opciones de respuesta que hay (entrevista del 4 de mayo).

\section{c. Tomar notas para completar una tarea}

En un grado menor, algunos estudiantes recurrieron a la estrategia de toma de notas para lograr culminar la tarea. Sin embargo, las dos estrategias anteriormente mencionadas fueron las más utilizadas por los usuarios.

E: Esas estrategias que tú mencionas, y las que mencionaste antes, eh, ¿forman ahora parte de tus esquemas mentales, o ya las habías utilizado antes en otros, en otros ejercicios?
F: No, pues yo nunca había interactuado con algo así por el estilo, nunca en ningún lado, en ningún estudio, entonces, eh, a partir de las estrategias, pues, es que lo que pasa es que yo escribí las estrategias en un cuaderno...

\section{Fijación de la atención en información textual y contextual específica}

En esta categoría agrupamos todas aquellas estrategias que de una u otra manera nos remitían a acciones específicas con respecto a la información textual y contextual, fuese oral o escrita. Se identificaron cinco categorías:

a. Centrar la atención en información clave (tema, palabras clave, adjetivos, información nuevadada, sufijos y prefijos)

En algunos otros casos, y a menor escala de frecuencia, los usuarios contestaron en las entrevistas haber utilizado palabras clave para identificar el contexto:

SR: Prestar atención en los momentos que nombraban personas, eventos y fechas importantes

( $\log$ del 11 de noviembre, pregunta 3).

Las frases como "presté más atención" reflejan claramente la manera en que los alumnos tienden a concentrarse en entender la información relevante. Vale la pena destacar que la estrategia de centrar la atención en información clave posee el primer lugar de frecuencia de uso en esta categoría y que en segundo lugar se encuentra hacer inferencias, estrategias que la propuesta didáctica explica en la sección de entrenamiento.

b. Identificar el contexto situacional o semántico

El contexto desempeñó un papel muy importante en la realización de las tareas. En algunos casos, la comprensión del contexto situacional fue el punto de partida para que el estudiante infiriera el significado de palabras desconocidas. En otros casos, por el contrario, los estudiantes infirieron el significado de palabras desconocidas para entender el contexto, es decir, las relaciones entre los participantes, sus intenciones, la trama de la historia y el papel que ellos como estudiantes debían desempeñar en dicha historia para utilizar el discurso apropiado 
para interactuar con los personajes de la historia de acuerdo con la caracterización que se hizo de ellos durante las misiones.

E: ¿Qué mecanismos o qué estrategias utilizabas para saber cuál era la respuesta correcta?

FM: Pues, primero prestándole atención a lo que ellos decían y teniendo en mente todo lo que ya había pasado durante el juego, y como siempre ha dicho la profesora: el contexto, es decir, las cosas que están en el contexto, a las palabras clave, lo de grupos semánticos y cosas de esas (entrevista del 26 de noviembre).

\section{c. Hacer inferencias con base en el contexto}

Los estudiantes afirmaron que, de acuerdo con las preguntas formuladas, tenían que inferir las respuestas y que en muchas ocasiones inferían también el significado de las palabras que no entendían, precisamente porque el contexto les permitía hacerlo. Este resultado es bastante significativo para el grupo de investigación, puesto que una de las tareas más difíciles a las que los estudiantes se enfrentan cuando están escuchando textos en inglés es la de hacer inferencias. A Journey to Britannia posibilita el desarrollo de esta estrategia, ya que el mismo juego proporciona las condiciones apropiadas para hacerlo, una ventaja que muchos ejercicios de escucha provenientes de otras fuentes no logran.

Otro aspecto que es importante mencionar es que, aunque los estudiantes no mencionan explícitamente qué proceso siguieron para inferir la respuesta correcta, como diseñadores del software podemos afirmar que era necesario que el usuario estableciera relaciones entre los valores de verdad de los enunciados, de acuerdo con las características establecidas para cada uno de los personajes en ese mundo creado a partir de la leyenda del Rey Arturo. De esta manera, además de entender el contenido textual de cada opción de respuesta, el estudiante debía descifrar las intencionalidades del personaje con quien interactuaba y tener en cuenta su misión como usuario para "producir"1 un discurso coherente con la misión que le había sido asignada.

1 El usuario debía escoger el enunciado apropiado para responder
E: ¿Cómo te pareció el nivel de lengua utilizado por los hablantes en el juego?

A: Buenísimo, porque, bueno, en cuanto a pronunciación le exige a uno mucho; en cuanto a, pues a vocabulario también, y es, está como muy bien estructurado porque al comienzo, como te digo, porque como Merlín es ciego, y como él es una persona de alto rango, por decirlo así, él habla despacio; pero después la bruja, como es más astuta, entonces habla más rápido, ¿no?, entonces como que está organizado el nivel de exigencia también de acuerdo al, al mismo argumento de... (entrevista del 26 de noviembre).

Ante la pregunta ¿cómo aplicó los conocimientos aprendidos en el módulo de estrategias?, dos estudiantes explicaron la manera en que el contexto les sirvió para descartar opciones y hallar la respuesta correcta. Ejemplo:

DR: En cada pregunta observar el contexto y de esta forma hallar la respuesta correcta, descartando las otras opciones que no me parecían satisfactorias para la respuesta ( $\log$ del 2 de mayo, pregunta 4).

\section{d. Analizar el registro del lenguaje (formal o informal)} Por otra parte, además de la habilidad que poseen los estudiantes para hacer inferencias, se puede observar otra estrategia de menor frecuencia, pero de innegable importancia debido a su relación estrecha con la inferencia: analizar el registro del lenguaje. Al respecto se puede leer el siguiente ejemplo:

E: Bueno, habían opciones que eran muy parecidas.

MD: Sí.

E: ¿Cómo hacías para escoger una y otra, una de la otra?

MD: Eh, precisamente tratando de remontarme a lo que era la historia, entonces habían dos, dos opciones: una que era digamos más, eh, como más, umm, una forma más decente de responder, una forma más formal; entonces lo que, lo que nos proponían era tratar de responder de la mejor manera posible; eso me di cuenta más o menos en

adecuadamente a la situación comunicativa planteada. 
dos, dos respuesta que tuve mal, que decían no eso no es una forma correcta de responder porque, $y$ le daba a uno las razones porque; entonces ya después uno podía (entrevista del 26 de noviembre).

Estos comentarios muestran que los estudiantes determinaron el contexto dado en el juego e infirieron el tipo de registro que tenían que utilizar, en este caso formal, para interactuar de manera apropiada con los personajes de la historia.

Para concluir el análisis de esta categoría, es importante hacer notar que en el planteamiento del entrenamiento en estrategias, en los juegos asociados a éstas y en el desarrollo de las misiones, se evidencia que en el procesamiento de la información se yuxtaponen los procesos ascendentes o bottomup (dirigidos por los datos) y los descendentes $o$ top-down, dirigidos conceptualmente, en los que las expectativas sobre lo que los datos sostendrán, nuestras experiencias pasadas y el conocimiento almacenado en la memoria a largo plazo tiñen inevitablemente nuestra interpretación de los datos.

Hemos visto que en este juego interactivo, el usuario realiza procesamiento ascendente cuando encuentra palabras cuyo significado desconoce y trata de inferir con base en el contexto lingüístico en el que se encuentra la palabra o recurre al diccionario. Y realiza procesos descendentes cuando utiliza su conocimiento previo sobre cómo proceder en situaciones similares a las presentadas en el juego para darles solución, por ejemplo, para tener en cuenta sus conocimientos pragmáticos acerca de la manera de interactuar con personas que encubren sus intenciones, con personas de diferente estatus social, entre otras.

\section{Uso de procesos metacognitivos}

En esta categoría se descubrió que, además de las estrategias de aprendizaje explicadas en el juego A Journey to Britannia, los usuarios recurrieron a sus propias estrategias e iniciativas para desarrollar las tareas. En esta categoría, agrupamos aquellas estrategias que surgían como iniciativa propia del estudiante para administrar su aprendizaje, su resolución de problemas de manera individual o recurriendo a otros. Toda esta experiencia e inicia- tiva de los usuarios por encontrar otras estrategias para enfrentarse a las tareas ratifica la posibilidad que la propuesta hipermedial ofrece a los alumnos de desarrollar en forma consciente una variedad de estrategias de aprendizaje diferentes a las que se proponen en el juego.

a. Aprender de los errores como herramienta del aprendizaje

YM: Repasar vocabulario y practicar más listening y en la próxima sesión pienso retomar los errores y tratar de tener mayor cuidado en no volver a cometerlos (log del 4 de mayo, pregunta 15).

b. Usar otros recursos para verificar la comprensión (diccionario, la profesora)

CM: De pronto, hay palabras difíciles que uno no tiene accesibilidad dentro del mismo programa para comprender el significado, ¿sí?, entonces toca acudir a la profesora, y si no está la profesora, pues al diccionario, entonces de pronto es un poquito complicado esa parte (entrevista del 11 de mayo).

c. Planear y monitorear el proceso de aprendizaje

T: ¿Qué estrategia utilizaste?

A: La que te digo, que hay vocabulario que uno puede unir al español, pero hay otro que no, que es totalmente distinto, entonces support, por ejemplo, es sustentar y no tanto ayudar a otra persona, que era lo que aparecía en esa pregunta, que esa persona está, que ayudaba de tal, de otra cosa (entrevista del 26 de noviembre).

d. Escuchar varias veces para confirmar la comprensión auditiva

T: Entonces ¿cómo hiciste, qué te dio resultado?

LS: me tocó volver a, o sea, yo daba la que creía, ¿no?, pero no era, entonces otra vez tenía que escuchar con mayor atención y tratar de tomar como el mensaje completo (entrevista del 11 de mayo).

Es importante anotar que dos estudiantes mencionaron cada uno una estrategia que les permitía aprender mejor y monitorear su aprendizaje. Aunque no se pueden tomar como categorías por su baja frecuencia, es bueno mencionarlas para completar la visión que tenemos sobre este grupo de estrate- 
gias, a saber: identificar el objetivo de la sección y manejar el tiempo.

\section{Aplicación de conocimientos previos y nuevos}

En esta categoría agrupamos las estrategias que nos permitían vislumbrar la aplicación de los conocimientos ya conocidos o aquellos que eran totalmente novedosos. Entre las estrategias relacionadas con conocimientos previos, algunos estudiantes reportaron haber utilizado las siguientes:

\section{a. Entender rasgos de pronunciación y supraseg- mentales}

En esta categoría, la estrategia con mayor frecuencia de uso fue ésta, pues hubo gran interés por utilizar los conocimientos previos y nuevos con referencia a la acentuación de palabra y oración, el ritmo marcado por grupos de pensamiento, la entonación, el tono y el acento de los hablantes nativos con el fin de entender mejor sus enunciados y sus intenciones. Los alumnos también destacaron el hecho de poder aprender pronunciación mediante los audios.

CM: (Aprendí) cómo la entonación que se le da a una oración puede ayudar a entender el mensaje que se quiere transmitir ( $\log$ del 23 de septiembre, pregunta 2).

b. Usar conocimiento declarativo lingüístico y no lingüístico previo y nuevo

Entre las estrategias relacionadas con conocimientos previos, algunos estudiantes reportaron haber utilizado su conocimiento de la lengua materna para comprender la lengua extranjera, L2. Igualmente, mencionan la conexión que realizan entre ese conocimiento de la lengua materna y el nuevo conocimiento en la lengua extranjera para ayudarse en la solución de las tareas.

E: ¿Qué te llamó más la atención?

DP: El manejo que le dan a los prefijos y a los sufijos, o sea, como la función que tienen, eso es como muy relativo al español, o sea como que uno... sí, por medio del significado que le dan uno lo relaciona bien con el español y lo entiende mejor en inglés (entrevista del 5 de mayo).

\section{c. Utilizar reglas de pronunciación (fonéticas)} Uno de los juegos en el módulo de estrategias buscaba mostrar a los estudiantes algunas reglas de pronunciación en relación con las consonantes que no se pronuncian en inglés.

DP: Me pareció muy útil lo de... lo de la pronunciación. Una parte que habla de la pronunciación. Cuándo hay que pronunciar, digamos en el caso de "mb", que no se pronuncia la "b". Para mí fue útil lo de la " $k$ ", al principio, eso lo sabe uno, pero entonces ahí como que lo afianza más, ¿no? (entrevista del 5 de mayo).

Durante este análisis hemos podido ver la manera en que los estudiantes utilizan las estrategias aprendidas y la de su propia invención para desarrollar las habilidades que menciona Nunan (1989) como esenciales para una escucha exitosa, como dividir el flujo de discurso en palabras y frases significativas, reconocer las categorías de las palabras, identificar la intencionalidad retórica y funcional de un enunciado o de las partes de un texto oral, interpretar el ritmo, el acento y la entonación con el fin de identificar el foco de la información y el tono emocional y actitudinal con que se producen los textos y extraer la información esencial de textos largos sin tener que comprender cada una de las palabras. Todo ello logrado mediante la planeación cuidadosa del diseño conceptual y gráfico del juego interactivo, el cual fue adecuado gracias a las sugerencias de los mismos estudiantes, en la fase de pilotaje.

\section{Conclusiones}

Esta investigación buscaba responder dos interrogantes, a saber: ¿cómo perciben los estudiantes las dos propuestas metodológicas desarrolladas en el ambiente hipermedial? Y ¿cómo se enfrentan los estudiantes de nivel intermedio y avanzado a realizar las tareas de escucha?

Como recordaremos, en la parte metodológica, con esta propuesta los docentes investigadores le apostamos a la integración de habilidades, al entrenamiento en estrategias de aprendizaje, al desarrollo de la competencia intercultural y a la promoción del trabajo autónomo como herramientas para apoyar 
el desarrollo de la comprensión auditiva. En la parte tecnológica, le apostamos a la interacción, a la retroalimentación, al poder del juego como elemento motivador en el aprendizaje, a la fascinación que producen las leyendas, al poder de la imagen para fijar información adquirida mediante otros modos de comunicación, a la evaluación inmediata de las respuestas que puede realizar el programa y a la calidad de la retroalimentación que puede ofrecer, a la variedad y a la personalización de la información que permite la hipertextualidad. En resumen, le apostamos al engranaje metodológico-tecnológico que permiten lograr los hipermedios.

El análisis de los datos nos indican que los estudiantes percibieron la propuesta metodológica como instrumento de práctica contextualizada e interactiva que genera motivación y facilita la comprensión, instrumento útil para la adquisición de conocimiento y desarrollo de habilidades, e instrumento que promueve el aprendizaje autónomo.

Por otro lado, pudimos determinar que los estudiantes se enfrentaron a las tareas de escucha utilizando, por una parte, y de una manera consciente, las estrategias en las que habían sido instruidos en la primera sección del juego y, por otra, sus propias estrategias. Como hemos podido apreciar mediante los ejemplos proporcionados, los estudiantes hicieron uso de las estrategias que se modelaron explícitamente, como razonamiento deductivo, comprensión de la idea general de manera rápida, uso de claves lingüísticas, grupos de pensamiento, transferencia lingüística, uso de palabras clave y grupos semánticos, pero llamándolas por nombres que les eran familiares, como podemos observar en el cuadro que se presenta al inicio del análisis de la segunda pregunta.

Creemos que si se fomenta un uso explícito de estrategias como las que hemos mostrado en este artículo, los estudiantes pueden enfrentarse a tareas de escucha con mayores herramientas y con menos aprensión hacia ellas.

Estos resultados también nos permiten afirmar que así como un hipermedia sustentado en los principios pedagógicos, metodológicos y tecnológicos que hemos tenido en cuenta en A Journey to Britannia tuvo un gran valor en los procesos de aprendizaje para los estudiantes de intermedio del Departamento de Lenguas de la Universidad Pedagógica Nacional, puede tenerlo también para otros estudiantes de universidades con programas de formación en lenguas y docentes en ejercicio y ser un modelo a seguir para profesores de inglés que quieran producir este tipo de ayudas mediadas por computador, con la colaboración de un equipo interdisciplinario. \1 


\section{Referencias bilbiográficas}

Arzaluz, S. (2005). La utilización del estudio de caso en el análisis local. Consultado en: http://lanic.utexas. edu/project/etext/colson/32/4araluz.pdf

Baquero, R. (1996). La Zona de Desarrollo Próximo y el análisis de las prácticas educativas. En Vigotsky y el aprendizaje escolar. (s. f.). Buenos Aires: Aique.

Barraza Macías, A. (2001). Constructivismo social: un paradigma en formación. Consultado en: http://www. psicologiacientifica.com/publicaciones/biblioteca/ articulos/ar-artbarra_01_3.htm

Brown, H. D. (1994). Teaching by principles. Nueva Jersey: Prentice Hall Regents.

Caillois, R. (1997). Los juegos y los hombres. La máscara y el vértigo. Bogotá: Fondo de Cultura Económica.

Faltis, C. (1997). Case study methods in researching language and education. En N. Hornberger y D. Corson (Eds.), Encyclopedia of Language and Education, vol. 8 Research Methods in Language and Education. The Netherlands, Kluwer Academic Publishers.

Freeman, D. (1998). Doing teacher research, from inquiry to understanding. Canadá: Heinle \& Heinle.

Gagné, E. (1985). The cognitive Psychology of school learning. Boston: Little, Brown and Company.

Genesee, F. y Upshur, J. (1996). Classroom-based evaluation. Cambridge: Cambridge University Press.

Heinich, M., Russell y Smaldino. (1999). Instructional media and technologies for learning. Columbus.

Holec, H. (1981). Autonomy in foreign language learning. Oxford: Pergamon.

Jonnassen, D. (2002). Technology as cognitive tools. Learners as designers. En Language learning and technology, 3, pp. 58-86.
Lamarca, M. J. Hipertexto: el nuevo concepto de documento en la cultura de la imagen. Tesis doctoral. Consultado en: http://www.hipertexto.info/documentos/indice.htm\#3

Merriam, S. (1998). Qualitative research and case study applications in education. San Francisco: Jossey-Bass.

McDonough, S. (2002). Applied linguistics in language education. Nueva York: Oxford University Press.

Nunan, D. (1989). Designing tasks for the communicative classroom. Melbourne: Cambridge Language Teaching Library.

Oxford, R. (1991). Language learning strategies. What every teacher should know. Newbury House.

Richards, J. (1990). The language teaching matrix: curriculum, methodology, and materials. Cambridge: Cambridge University Press.

Richards, J. y Rodgers, T. (2002). Approaches and methods in language teaching. Cambridge: Cambridge University Press.

Skehan, P. (1998). A cognitive approach to language learning. Oxford: Oxford University Press.

Tudor, I. (2001). The dynamics of language classroom. Cambridge: Cambridge University Press.

Warschauer, M. y Healey, D. (1998). Computers and language learning: an overview. Language Teaching, 31, pp. 57-71.

Wells, G. (1995). Language and the inquiry oriented curriculum. Ontario: The Ontario Institute for Studies in Education.

Williams, M., Robert, B. (1997). Psychology for teachers. Cambridge: Cambridge University Press.

White, R. (1988). The ELT curriculum: design, innovation, and management. Estados Unidos: Wiley-Blackwell. 